\title{
A Comparative Study of Intramedullary Nailing and Minimally Invasive Percutaneous Plate Osteosynthesis for Type A Tibial Fracture Healing
}

\author{
Huang Jianjun $^{1} \quad$ Su Songsen $^{1} \quad$ Liu Yongqing ${ }^{1} \quad$ Yu Haibin ${ }^{1, *}$
}

\author{
${ }^{1}$ Department of Orthopedics, Binhaiwan Central Hospital of Dongguan, Dongguan, Guangdong, China, 523900 \\ *Haibin Yu is the corresponding author. https://orcid.org/0000-0001-6983-6939.
}

\begin{abstract}
Article History Received 10 July 2020 Accepted 25 July 2020 Published 30 September 2020 Cite this Article Huang Jianjun, Su Songsen, Liu Yongqing, Yu Haibin. A Comparative Study of Intramedullary Nailing and Minimally Invasive Percutaneous Plate Osteosynthesis for Type A Tibial Fracture Healing [J].Medical Research, 2020.2(3):14-19, http://dx.doi.org/10.6913/MRHK.202009_2(3).0003 Copyright @ 2020 Creative Publishing Co., Limited. All rights reserved. Email:mrhk26640333@gmail.com.
\end{abstract}

\section{ABSTRACT}

Objective This study aimed to retrospectively compare the efficacy of intramedullary nailing and minimally invasive percutaneous plate osteosynthesis (MIPPO) on the fracture healing of patients with type A tibial fractures.

Methods Patients who had been diagnosed with type A tibial fracture (AO classification method) and treated with minimally invasive surgery in our hospital from January 2016 to January 2019 were included in the study. There were 21 patients in the MIPPO group and 27 patients in the intramedullary nailing group. The operation time, intraoperative blood loss, surgical incision length, callus formation time, and fracture healing time of the two groups were analyzed and compared.

Results In the intramedullary nailing group, the callus formation time and the fracture healing time were shorter than those in the MIPPO group $(\mathrm{P}<0.005)$. Their operation time, intraoperative blood loss, and the surgical incision length were comparable between the two groups.

Conclusions For patients with type A tibial fracture and poor wound tissue conditions, intramedullary nailing fixation treatment may be a better choice than MIPPO. Because in patients receiving intramedullary nailing treatment, the callus formation time and fracture healing time are short, and functional exercises can be performed soon after the surgery, which can reduce the occurrence of related complications.

Keywords Tibial fractures; Intramedullary nailing; MIPPO; Callus formation; Fracture healing time

\section{INTRODUCTION}

Tibia fracture is one of the most common fractures, which is mainly caused by high-velocity trauma. Due to the special anatomical structure of the tibia, such as less nourishing blood vessels, poor blood supply, less soft tissue coverage, etc., the possibility of delayed union or nonunion after fracture is higher, and compartment syndrome may occur. There are many ways to treat tibial fractures, such as plaster external fixation, external fixation, plate internal fixation and intramedullary nailing fixation ${ }^{[1,2]}$. However, traditional open reduction and internal fixation surgery have many shortcomings, including large surgical 
Huang Jianjun, et.,al. A Comparative Study of Intramedullary Nailing and Minimally Invasive Percutaneous Plate Osteosynthesis for Type A Tibial Fracture Healing Medical Research ISSN 2664-0333 eISSN 2664-0341 Volume 2 Issue 3 http://dx.doi.org/10.6913/MRHK.202009_2(3).0003

incisions leading to extensive periosteal dissection, serious damage to soft tissues and blood supply, and related complications after surgery. Therefore, the current anatomical tibial intramedullary nailing and MIPPO emerge as the preferred approaches for type A tibial fractures.

In clinical practice, for patients with tibial fractures, weight-bearing functional exercises can only be performed after X-rays show callus formation. However, there are few studies on the comparison of callus formation time and final healing time of tibia intramedullary nailing and MIPPO.

This study retrospectively analyzed the monthly X-rays of patients undergoing minimally invasive surgery for type A tibial fractures in our hospital and compared the effects of tibial intramedullary nailing fixation and MIPPO in various aspects.

\section{MATERIALS AND METHODS}

\section{Patient information}

Patients who were diagnosed with type A tibial fractures (AO classification method) and treated with minimally invasive surgery in our hospital from January 2016 to January 2019 were selected. The patients were divided into two groups according to different surgical methods. The MIPPO group (Group A; n=21) included 10 males and 11 females. Their age ranged from 14 to 55 years old. The tibial intramedullary nailing fixation group (Group B; n=27) included 15 males and 12 females, and their ages ranged from 23 to 70 years. Patients with type B or C fractures, open fractures, pathological fractures, or old fractures, combined with nerve or vascular injury, incomplete data, and no monthly regular follow-up were excluded.

\section{Intervention}

After the operation and during the monthly follow-up, all patients received X-rays of the tibia and fibula in the supine position. The pictures were then downloaded from the PACS reading system. The Photoshop 7.0 image processing system was used to adjust the grayscale of the picture (so that it is suitable for visual analysis) and to adjust the picture size (so that the image details are highlighted). The Photoshop 7.0 image processing system was also used for imaging processing to reduce reading errors.

\section{Observation parameters}

The data including callus formation time (week), fracture healing time (week), operation time (min), intraoperative blood loss $(\mathrm{ml})$, and surgical incision length $(\mathrm{cm})$ of patients between the two groups were collected.

\section{Statistical analysis}

The SPSS 21.0 statistical analysis software, paired t-test analysis was used for statistical analysis. A p-value less than 0.05 indicated that the result was considered statistically significant.

\section{RESULT}

All patients completed the operation and recovered without any serious complications. The key parameters including callus formation time, fracture healing time, operation time, intraoperative blood loss and surgical incision length values in patients between the two groups are shown in Table 1. The callus 
Huang Jianjun, et.,al. A Comparative Study of Intramedullary Nailing and Minimally Invasive Percutaneous Plate Osteosynthesis for Type A Tibial Fracture Healing Medical ResearchＩSSN 2664-0333 eISSN 2664-0341Ｖolume 2 Issue 3 http://dx.doi.org/10.6913/MRHK.202009_2(3).0003

formation time and fracture healing time of Group A patients were significantly shorter than those of Group B patients, suggesting that compared to MIPPO, intramedullary nailing fixation may be superior in patients' recovery. No significant difference was observed regarding the operation time and intraoperative blood loss between the two groups, while the surgical incision length in patients receiving intramedullary nailing fixation was longer than that in patients receiving MIPPO.

Table 1. Key parameters between the two groups

\begin{tabular}{ccccc}
\hline Parameters & Group A & Group B & T Value & P Value \\
\hline Callus formation time (week) & $11.1 \pm 2.8$ & $7.8 \pm 2.7$ & 3.288 & 0.004 \\
Fracture healing time (week) & $34.5 \pm 3.9$ & $29.9 \pm 6.5$ & 5.371 & 0.003 \\
Operation time (min) & $125.5 \pm 35.5$ & $121.1 \pm 43.9$ & -0.440 & 0.665 \\
Intraoperative blood loss (ml) & $25.4 \pm 10.7$ & $31.0 \pm 11.7$ & -1.787 & 0.089 \\
Surgical incision length (cm) & $4.7 \pm 1.1$ & $5.3 \pm 1.2$ & -3.081 & 0.006 \\
\hline
\end{tabular}

The representative X-ray images of patients receiving MIPPO and intramedullary nailing fixation were shown in Figure 1 and Figure 2.
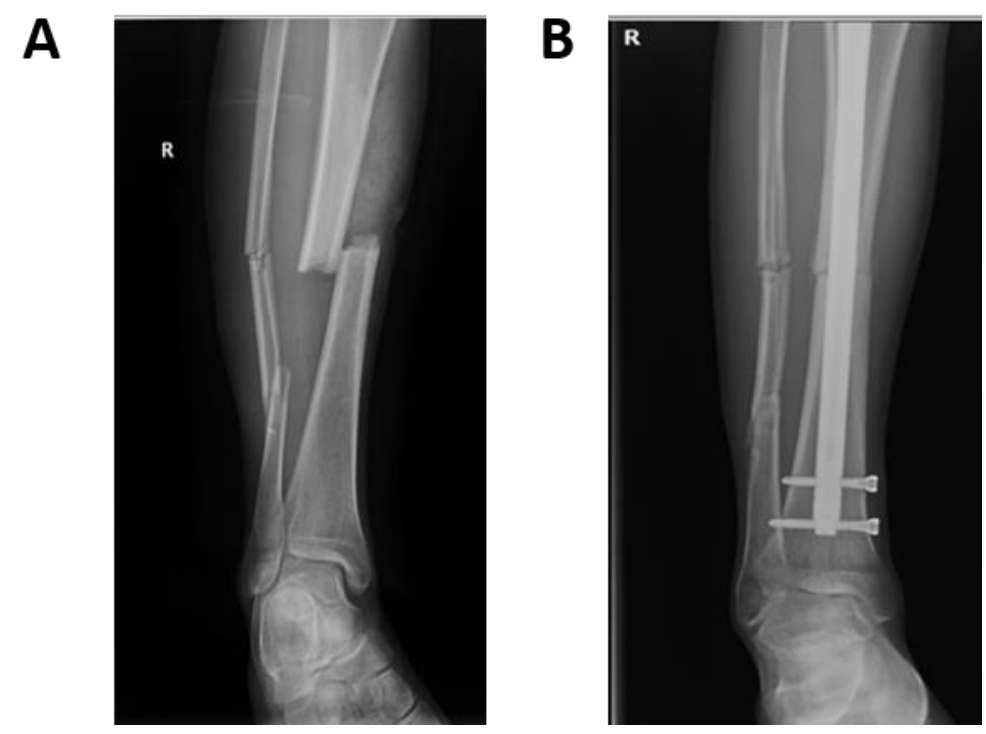

Figure 1: Representative $\mathrm{X}$-ray images of tibia fracture patients receiving intramedullary nailing fixation. (A) The tibia fracture before the operation is shown. (B) Callus appeared 7 weeks after the operation.

\section{DISCUSSION}

Tibia fractures are one of the most common long bone injuries, usually caused by high-energy violence. In view of the relative lack of attached soft tissue and blood supply in the special anatomical structure of the tibia, improper treatment may lead to fracture nonunion or delayed union ${ }^{[3]}$. In addition, patients with tibial fractures often suffer from severe pain, swelling and movement disorders at the fracture site, so early and appropriate surgery plays an important role in alleviating patients' symptoms and improving their motor functions ${ }^{[4]}$. 

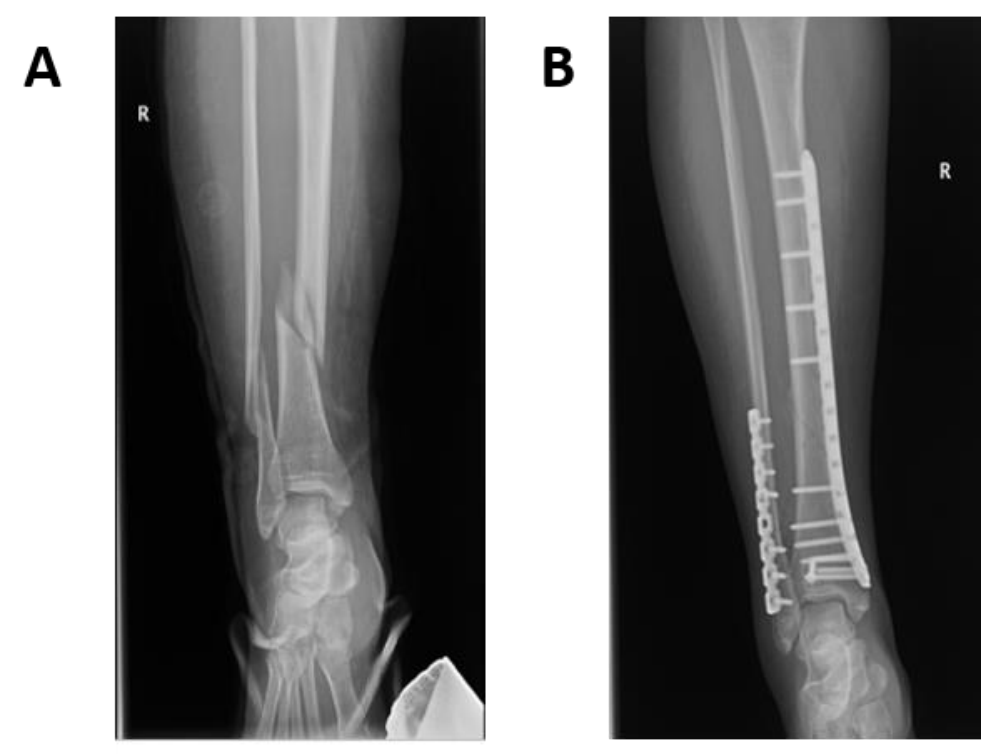

Figure 2: Representative X-ray images of tibia fracture patients receiving MIPPO. (A) The tibia fracture before the operation is shown. (B) Callus appeared 10 weeks after the operation.

For type A fractures, anatomical tibial intramedullary nailing and MIPPO are the preferred choices for treatments. In this study, patients with type A tibial fractures in our hospital were selected and treated with anatomical tibial intramedullary nailing or MIPPO. The results showed that the fracture healing time and callus formation time of the intramedullary nailing group were significantly shorter than that of the MIPPO group, suggesting that the intramedullary nailing could help accelerate the growth of the callus at the fractured site of the tibia. This study showed that tibial intramedullary nailing treatment of tibial fractures was associated with early formation of callus at the fracture site.

Biomechanical studies of fractures have found that the reduction in axial stiffness of internal fixation devices could be beneficial to fracture healing ${ }^{[5,6]}$. Bottlang et al. ${ }^{[7]}$ believed that the stress shielding effect of the fracture end produced by the high rigidity of the steel plate will cause the growth of the callus of the fracture and may lead to the nonunion of the fracture. Gicquel et al. ${ }^{[8]}$ held the view that the structure of the internal nailing can stimulate the periosteal response and promote the formation of callus, and the periosteum plays an important role in the process of fracture healing. In addition, the placement of a steel plate for tibial fractures may have a "stress block" effect, resulting in slower callus growth and even delayed fracture healing ${ }^{[9,10]}$. Intramedullary nailing uses central fixation, and the stress distribution in the fracture area is relatively uniform, and there is no need to harass the periosteum and soft tissue around the fracture during the treatment, which protects the blood supply to the fracture end to the greatest extent and can accelerate the formation of callus ${ }^{[11]}$. Moreover, Matuszewski et al. ${ }^{[12]}$ showed that compared with the distal fixation plate, the probability of nonunion after intramedullary nailing fixation was lower.

In clinical practice, we usually use imaging examinations to determine the timing of the patient's complete weight-bearing based on the formation of the callus. In our research, we found that patients using intramedullary nailing need a shorter time for callus growth, which can be used for earlier weight-bearing activities or postoperative functional exercises. This is consistent with studies by Qin et al. ${ }^{[13]}$ and others. In 
the study, it is believed that although patients undergoing MIPPO and intramedullary nailing treatment can achieve satisfactory results, the intramedullary nailing fixation group has earlier weight-bearing time, and the knee and ankle joints move after surgery. In terms of ability, patients in the intramedullary nailing group performed better. Moreover, in the study by Gross et al. ${ }^{[14]}$, patients with intramedullary nailing fixation could perform functional rehabilitation exercises at an earlier stage, and early weight-bearing activities also promote fracture healing due to stress stimulation, forming a virtuous cycle. Early functional exercise can effectively prevent complications such as muscle atrophy, joint stiffness and thrombosis, and can increase patient compliance,

In the fluoroscopy, if the residual angle is found to be greater than $5^{\circ}$ on the anamorphic surface or the coronal surface, it can be regarded as malunion ${ }^{[15]}$. Gicquel et al. ${ }^{[8]}$ believed that patients with tibial fractures treated with intramedullary nailing have a certain chance of lower limb angulation deformity. However, we did not find patients with obvious deformities in the study. Chen et al. ${ }^{[16]}$ believed that because the intramedullary nailing needs to be inserted from the front of the knee joint, there may be joint adhesions and patella impingement after the operation, which may lead to limited joint activities and affect the function of the knee joint. However, Zhu et al. ${ }^{[17]}$ and others believed that this situation could be avoided by changing the approach. Fang et al. ${ }^{[18]}$ showed that because of the large medullary cavity of the distal tibia, the intramedullary nailing may be offset after inserting it. This situation often requires the assistance of blocking nailing technology, thereby increasing the operation time.

This study has several limitations. First, the follow-up interval of patients was once a month. If the number of follow-ups of patients could be increased in the first three months, the conclusion may be more precise. Second, we did not consider other factors that will affect the callus formation time, such as the patient's nutritional status, whether the patient had osteoporosis, etc. Third, during the follow-up period, the patient's weight-bearing exercise and full-body exercise were not included in the analysis.

Although intramedullary nailing and MIPPO have advantages and disadvantages, for patients with type A fractures and poor wound healings, intramedullary nailing treatment may be a better choice. Our study shows that compared with MIPPO, the callus formation time and fracture healing time of intramedullary nailing treatment are shorter, and patients can perform functional exercises earlier, which helps prevent complications such as muscle atrophy, joint stiffness, and thrombosis disease.

\section{ACKNOWLEDGEMENT}

None.

\section{STATEMENT}

There is no conflict of interest in this article.

\section{REFERENCES}

1 Weiguo Zhu. Clinical Effects of Expert Tibial Nail in Treating Distal Tibial Fracture. Journal of Tissue Engineering and Reconstructive Surgery. 2013, 9(05):276-278.

2 Haibin Sun. Treatment of distal tibial fractures with minimally invasive percutaneous internal fixation. Guide of 
Huang Jianjun, et.,al. A Comparative Study of Intramedullary Nailing and Minimally Invasive Percutaneous Plate Osteosynthesis for Type A Tibial Fracture Healing Medical Research ISSN 2664-0333 eISSN 2664-0341 Volume 2 Issue 3 http://dx.doi.org/10.6913/MRHK.202009_2(3).0003

China Medicine. 2013, 11(28):393-394.

3 Dachuan Sun, Chunlin Hu, Hao Sun et al. The effect of minimally invasive osteosynthesis combined with LCP in the treatment of distal tibia fibula fracture. Journal of Hunan Normal University (Medical Science). 2017, 14(02):173-176.

4 Qing-quan Chen, Zhi-hong Zhang, Wan-ming Wang. Clinical application of percutaneous minimally invasive locking compression plate on distal tibial fractures. Orthopedic Journal of China. 2012, 20(14):1295-1298.

5 Claes L, Blakytny R, Gockelmann M, et al. Early dynamization by reduced fixation stiffness does not improve fracture healing in a rat femoral osteotomy model. J Orthop Res. 2009, 27(1): 22-27.

6 Bottlang M, Doornink J, Byrd G D, et al. A nonlocking end screw can decrease fracture risk caused by locked plating in the osteoporotic diaphysis. J Bone Joint Surg Am. 2009, 91(3): 620-627.

7 Bottlang M, Doornink J, Fitzpatrick D C, et al. Far cortical locking can reduce stiffness of locked plating constructs while retaining construct strength. J Bone Joint Surg Am. 2009, 91(8): 1985-1994.

8 Gicquel P, Giacomelli M, Basic B, et al. Problems of operative and non-operative treatment and healing in tibial fractures. Injury. 2005, 36(1): S44-S50.

9 Kuhn S, Greenfield J, Arand C, et al. Treatment of distal intraarticular tibial fractures: A biomechanical evaluation of intramedullary nailing vs. angle-stable plate osteosynthesis. Injury. 2015, 46 Suppl 4: S99-S103.

10 Seyhan M, Unay K, Sener N. Intramedullary nailing versus percutaneous locked plating of distal extra-articular tibial fractures: a retrospective study[J]. Eur J Orthop Surg Traumatol. 2013, 23(5): 595-601.

11 Mohammad L M, Messegee J, Chohan M O, et al. Fluoroscopic Cranial Radiation Exposure in Spine Surgery: A Prospective Single-Center Evaluation in Operating Room Personnel. Int J Spine Surg. 2019, 13(1): $28-32$.

12 Matuszewski P E, Mehta S. Fracture consolidation in a tibial nonunion after revascularization: a case report[J]. J Orthop Trauma. 2011, 25(2): e15-e20.

13 Wen-jie Qin, Cheng-ming Zhu, Hao Wu et al. Comparison of two internal fixation methods for multiple segment tibial shaft fractures. Orthopedic Journal of China. 2020, 28(6):496-500.

14 Gross S C, Galos D K, Taormina D P, et al. Can Tibial Shaft Fractures Bear Weight After Intramedullary Nailing? A Randomized Controlled Trial[J]. J Orthop Trauma. 2016, 30(7): 370-375.

15 Teitz C C, Carter D R, Frankel V H. Problems associated with tibial fractures with intact fibulae[J]. J Bone Joint Surg Am. 1980, 62(5): 770-776.

16 Gang Chen, Ming-quan Qian, Guo-xing Zhu et al. Percutaneous closed reduction locking compression plate, percutaneous closed reduction interlocking intramedullary nail and open reduction plate in the treatment of tibial fracture: comparison of biostability. Journal of Clinical Rehabilitative Tissue Engineering Research. 2014, 18(44):7151-7156.

17 Long-zhang Zhu, Feng Xue, Ben-song Han. Knee function comparison and histological evaluation of the tibial shaft after intramedullary nail fixation. Journal of Clinical Rehabilitative Tissue Engineering Research. 2011, 15(43):8079-8082.

18 Wei Fang, Zhaolong Ma, Wengui Yang et al. Biomechanical study of combined blocking screws and intramedullary nails to treat distal tibial fractures. Chinese Journal of Bone and Joint Injury. 2014, 29(04):359-361. 